

\title{
On the Picard number of K3 surfaces over number fields
}

\author{
François Charles
}

\begin{abstract}
We discuss some aspects of the behavior of specialization at a finite place of Néron-Severi groups of K3 surfaces over number fields. We give optimal lower bounds for the Picard number of such specializations, thus answering a question of Elsenhans and Jahnel. As a consequence of these results, we show that it is possible to compute explicitly the Picard number of any given K3 surface over a number field.
\end{abstract}

\section{Introduction}

This paper deals with two questions concerning the arithmetic and the geometry of $\mathrm{K} 3$ surfaces. Let $X$ be a polarized $\mathrm{K} 3$ surface over a number field $k$, and let $\mathfrak{p}$ be a finite place of $k$ where $X$ has good reduction. Denote by $X_{\mathfrak{p}}$ the special fiber of a smooth model of $X$ over the ring of integers of $k_{\mathfrak{p}}$. Denote by $\bar{X}$ the base change of $X$ to an algebraic closure of $k$, and by $\bar{X}_{\mathfrak{p}}$ the base change of $X_{\mathfrak{p}}$ to the residue field of $\mathfrak{p}$. Specialization of divisors induces a specialization map between the Néron-Severi groups of $\bar{X}$ and $\bar{X}_{\mathfrak{p}}$.

Question 1. What can be said about the specialization map

$$
s p: N S(\bar{X}) \rightarrow N S\left(\bar{X}_{\mathfrak{p}}\right) ?
$$

A standard argument using the cycle class map and the smooth base change theorem shows that this specialization map is always injective up to torsion. We are here interested in the defect of surjectivity. This problem has been featured prominently in [Bogomolov, Hassett and Tschinkel 2011] and [Li and Liedtke 2012].

The second question is the following. Recall that the Picard number of a variety is by definition the rank of its Néron-Severi group.

Question 2. Given a projective embedding of $X$, is it possible to compute the geometric Picard number of $X$ ?

MSC2010: primary 14J28; secondary 14C22, 14G25, $11 \mathrm{G} 35$.

Keywords: K3 surfaces, Picard number, Néron-Severi group. 
This question is raised by Shioda [1981].

Using the Weil conjectures [Deligne 1974], it is possible to compute the Picard numbers of smooth projective varieties over finite fields, at least in the case the Tate conjecture holds. Indeed, counting points in sufficiently many extensions of the base field, one can compute the characteristic polynomial of the Frobenius acting on the second étale cohomology group and determine the multiplicity of 1 as an eigenvalue. If the Tate conjecture holds, this multiplicity is equal to the Picard number.

In characteristic 0, Question 2 is more difficult. In particular, the first explicit example of a K3 surface over a number field with Picard rank 1 has been recently given by van Luijk [2007]. Van Luijk's method provides a link between both questions. Indeed, it proceeds by computing Picard numbers at sufficiently many finite places in order to get information over the field of definition. In the past few years, the problem of computing Picard numbers of K3 surfaces has been featured for instance in the work of Elsenhans and Jahnel [2008a; 2008b] with recent geometric applications in the work of Hassett and Várilly-Alvarado [2013] and Várilly [Hassett et al. 2011]. Recent work of Hassett, Kresch and Tschinkel [2013] tackles this question in some cases.

With this approach, one of the main problems is finding finite places $\mathfrak{p}$ such that the specialization map (1) is as close to being surjective as possible, that is, such that $\rho\left(\bar{X}_{\mathfrak{p}}\right)$ is as small as possible.

Note that the situation in this mixed characteristic setting is in stark contrast with the case of equal characteristic 0 . Indeed, for K3 surfaces defined over function fields over $\mathbb{C}$ or $\overline{\mathbb{Q}}$, most specializations induce isomorphisms at the level of the Néron-Severi group. This is a consequence of Baire's theorem over $\mathbb{C}$ (see for instance [Voisin 2002, Chapter 13]) and of the Hilbert irreducibility theorem over $\overline{\mathbb{Q}}$ as was first noticed by Terasoma [1985; André 1996, Theorem 5.2(3)]. A different approach to this problem can be found in [Maulik and Poonen 2012].

On the other hand, over finite fields, there are obstructions for the map (1) to be surjective as was first noticed by Shioda [1981; 1983]. Indeed, it is a consequence of the Tate conjecture that the geometric Picard number of a K3 surface over a number field is always even; see for instance [de Jong and Katz 2000]. This striking fact has been recently used in a surprising way by Bogomolov, Hassett and Tschinkel [2011] and Li and Liedtke [2012] to prove that any complex K3 surface with odd Picard rank contains infinitely many rational curves.

In this paper, we describe the Shioda-type obstructions that can prevent the map (1) from being surjective, and we give optimal lower bounds for the Picard number of the specialization. One of our results is that Hodge theory can force the existence of such obstructions even when the Picard number is even; see Theorem 1(2) below. 
Let $X$ be a K3 surface over a number field $k$, and choose a complex embedding of $k$. Let $\rho$ be the geometric Picard number of $X$, and for any finite place $\mathfrak{p}$ of $k$ where $X$ has good reduction, let $\rho_{\mathfrak{p}}$ be the geometric Picard number of $X_{\mathfrak{p}}$. Note that we always have

$$
\rho_{\mathfrak{p}} \geq \rho .
$$

We need to control the Hodge theory of $X_{\mathbb{C}}$. Let $T$ be the orthogonal complement of $N S\left(X_{\mathbb{C}}\right)$ in the singular cohomology group $H^{2}\left(X_{\mathbb{C}}, \mathbb{Q}\right)$ with respect to cupproduct. The space $T$ is a sub-Hodge structure of $H^{2}\left(X_{\mathbb{C}}, \mathbb{Q}\right)$. Let $E$ be the algebra of endomorphisms of $T$ that respect the Hodge structure. Zarhin [1983] shows that $E$ is either a totally real field or a CM field.

The following result can be considered as a number field analog of the specialization results over function fields mentioned above:

Theorem 1. Let $X, T$ and $E$ be as above.

(1) If $E$ is a CM field or the dimension of $T$ as an E-vector space is even, then there exist infinitely many places $\mathfrak{p}$ of good reduction such that $\rho_{p}=\rho$. Furthermore, after replacing $k$ by a finite extension, this equality holds for a set of places of density 1.

(2) Assume $E$ is a totally real field and the dimension of $T$ as an $E$-vector space is odd.

Let $\mathfrak{p}$ be a finite place of $k$ where $X$ has good reduction. Assume that the characteristic of the residue field of $\mathfrak{p}$ is at least 5 . Then

$$
\rho_{\mathfrak{p}} \geq \rho+[E: \mathbb{Q}] .
$$

The equality $\rho_{\mathfrak{p}}=\rho+[E: \mathbb{Q}]$ is satisfied for infinitely many places $\mathfrak{p}$ of good reduction. Furthermore, after replacing $k$ by a finite extension, this equality holds for a set of places of density 1 .

Remark 2. Note that if $\rho$ is odd, the dimension of $T$ over $\mathbb{Q}$ is odd; hence, $X$ satisfies the assumptions of the second part of the theorem.

Remark 3. Elsenhans and Jahnel [2012] ask whether, with notation as in the theorem, there exists $\mathfrak{p}$ such that $\rho_{\mathfrak{p}}-\rho \leq 1$. The result above shows that it is not the case if $E$ is a totally real field of degree at least 2 over $\mathbb{Q}$ such that the dimension of $T$ over $E$ is odd. This is however true in all other cases.

This result shows that the Picard number can be forced to jump in specializations even when the Picard number of $X$ is even. Using the method of Li and Liedtke [2012], we get the following corollary:

Corollary 4. Let $X$ be either a K3 surface of Picard rank 2 with $E$ a totally real field of degree 4 or a K3 surface of Picard rank 4 with $E$ a totally real field of even degree. Then $X$ contains infinitely many rational curves. 
There exist such K3 surfaces by [van Geemen 2008, Section 3], and they give new examples of K3 surfaces with infinitely many rational curves. Note that complex K3 surfaces of Picard rank different from 2 and 4 are known to contain infinitely many rational curves by [Li and Liedtke 2012].

The second main result of this paper is a solution to Question 2. Recall that the method of van Luijk [2007] to prove that a K3 surface $X$ over $\mathbb{Q}$ has Picard number 1 was to first find two primes $p$ and $q$ of good reduction such that $X$ specializes to a K3 surface of Picard number 2 modulo $p$ and $q$. If the discriminant of the Néron-Severi lattices modulo $p$ and $q$ differ by a nonsquare factor, van Luijk shows that this implies that $X$ has Picard number 1 .

By Remark 3, there are cases where we cannot expect van Luijk's method to work directly for all K3 surfaces of rank 1. However, the second part of Theorem 1 can be used to show that reduction at finite places does indeed give enough information to compute Picard numbers over number fields.

This gives a theoretical explanation to the computations in [van Luijk 2007; Elsenhans and Jahnel 2008a; 2008b; Hassett et al. 2011; Hassett and VárillyAlvarado 2013].

Theorem 5. There exists an algorithm that, given a projective $K 3$ surface $X$ over a number field, either returns its geometric Picard number or does not terminate.

If $X \times X$ satisfies the Hodge conjecture for codimension- 2 cycles, then the algorithm applied to $X$ terminates.

Remark 6. In case the algorithm terminates, it is possible to compute the Picard number over the base field by searching for divisors spanning the geometric NéronSeveri group with rational coefficients and taking invariants under the action of the Galois group.

Remark 7. Let $X$ be a $\mathrm{K} 3$ surface over $\mathbb{C}$. With the notation of Theorem 1 , $X \times X$ satisfies the Hodge conjecture if and only if the field $E$ acts by algebraic correspondences. By [André 1996], this would be a consequence of the standard conjectures. Mukai [2002] has announced a proof in the case $E$ is a CM field.

Remark 8. The proof of the theorem actually shows that the only case where the algorithm would not terminate is, with the notation of Theorem 1, if $E$ is a totally real field that does not act on $H^{2}(X, \mathbb{Q})$ by algebraic correspondences and $T$ is of odd dimension as a vector space over $E$.

In particular, the algorithm always terminates for surfaces with $E=\mathbb{Q}$.

While we only consider K3 surfaces in this paper, some of the methods we consider have a wider range of applications. Assuming general conjectures on algebraic cycles, it is a general fact that the Mumford-Tate group associated to the second cohomology group of a variety controls specialization of Néron-Severi 
groups in a fashion that is similar to the way the monodromy representation appears in [André 1996; Maulik and Poonen 2012]. The multiplicity of the weight 0 in the corresponding representation is what forces the Picard number to jump after specialization. This is related to algorithmic computations of Néron-Severi groups as in our paper.

For K3 surfaces, the work of Zarhin [1983] and Tankeev [1990; 1995] allows us to give precise and unconditional results. The results of our paper conjecturally hold for varieties with $h^{2,0}=1$. It seems likely that one can prove them unconditionally for holomorphic symplectic varieties by extending the work of Tankeev cited above.

In Section 2, we recall results of Zarhin and Tankeev on the second cohomology group of a K3 surface. This allows us to prove Theorem 1 in Section 3. Section 4 is devoted to discriminant computations that will allow us to prove Theorem 5 in Section 5 .

\section{Algebraic monodromy groups of $\mathrm{K3}$ surfaces over number fields}

The results of this section are mostly contained in the work of Zarhin and Tankeev. After recalling some preliminary material, we describe the algebraic monodromy group of a K3 surface defined over a number field.

2.1. Mumford-Tate groups and the Mumford-Tate conjecture. Let $\mathbb{S}$ be the Deligne torus, that is, the algebraic group over $\mathbb{R}$ defined as

$$
\mathbb{S}=\operatorname{Res}_{\mathbb{C}} / \mathbb{R} \mathbb{G}_{m} .
$$

Let $H$ be a finite-dimensional vector space over $\mathbb{Q}$. Giving a Hodge structure on $H$ is equivalent to giving an action of $\mathbb{S}$ on $H_{\mathbb{R}}=H \otimes \mathbb{R}$.

Definition 9. Let $H$ be a rational Hodge structure. The Mumford-Tate group of $H$ is the smallest algebraic subgroup $M T(H)$ of $G L(H)$ such that $M T(H)_{\mathbb{R}}$ contains the image of $\mathbb{S}$ in $G L\left(H_{\mathbb{R}}\right)$.

We refer to [Deligne et al. 1982, Chapter I] for general properties of MumfordTate groups. Since $\mathbb{S}$ is connected, this definition implies that Mumford-Tate groups are connected. Note that the Mumford-Tate group of a polarized Hodge structure is reductive.

Let $i$ and $j$ be nonnegative integers, and consider the Hodge structure

$$
V=H^{\otimes i} \otimes\left(H^{*}\right)^{\otimes j}
$$

The Mumford-Tate group $M T(H)$ acts on $V$. If $v$ is a Hodge class in $V$, then the line $\mathbb{Q} v$ is globally invariant under the action of $M T(H)$. Conversely, it follows from Chevalley's theorem on affine groups that $M T(H)$ is the largest algebraic 
subgroup of $G L\left(H_{\mathbb{C}}\right)$ that leaves all such lines globally invariant [Deligne et al. 1982, Chapter I, Proposition 3.4].

We now turn to the $\ell$-adic theory. General results can be found in [Serre 1981]. Let $k$ be a number field, and fix an algebraic closure $\bar{k}$. Let $X$ be a smooth projective variety over $k$, and denote by $\bar{X}$ the variety $X \times{ }_{\operatorname{Spec} k} \operatorname{Spec} \bar{k}$. Fixing a prime number $\ell$, we can consider the étale cohomology group $H^{i}\left(\bar{X}, \mathbb{Q}_{\ell}\right)$ for some integer $i$. Let $\rho_{\ell}$ denote the continuous representation

$$
\rho_{\ell}: G_{k} \rightarrow G L\left(H^{i}\left(\bar{X}, \mathbb{Q}_{\ell}\right)\right)
$$

of the absolute Galois group $G_{k}$ of $k$. The image of $\rho_{\ell}$ is an $\ell$-adic Lie group.

Definition 10. With notation as above, let $G_{\ell}$ be the Zariski closure of the image of $\rho_{\ell}$ in the algebraic group $G L\left(H^{i}\left(\bar{X}, \mathbb{Q}_{\ell}\right)\right)$. The algebraic group $G_{\ell}$ is called the algebraic monodromy group associated to the Galois representation $\rho_{\ell}$.

Note that replacing $k$ by a finite extension replaces $G_{\ell}$ by an open subgroup of finite index. In particular, the identity component of the algebraic monodromy group does not depend on the choice of a field of definition for $X$.

General conjectures on algebraic cycles give important information on MumfordTate and algebraic monodromy groups. In particular, the latter are expected to be reductive. The expected relationship between those two groups is described by the Mumford-Tate conjecture as follows; see [Serre 1981].

Conjecture 11. Let $k$ be a number field, and fix a complex embedding of $k$. Let $X$ be a smooth projective variety over $k$.

Let $G_{\ell}$ be the algebraic monodromy group associated to the étale cohomology group $H^{i}\left(X_{\mathbb{C}}, \mathbb{Q}_{\ell}\right)$ for some prime number $\ell$, and let $G_{\ell}^{\circ}$ be its identity component. Then there is a canonical isomorphism

$$
G_{\ell}^{\circ} \simeq M T\left(H^{i}\left(X_{\mathbb{C}}, \mathbb{Q}\right)\right)_{\mathbb{Q}_{\ell}} .
$$

The Mumford-Tate conjecture is implied by the conjunction of the Tate and Hodge conjectures. A lot of work has been done in its direction in the case of abelian varieties [Serre 1998; Pink 1998; Vasiu 2008].

In this paper, we will focus on the case of K3 surfaces, where the MumfordTate conjecture holds. However, an important part of our method concerning specialization of Néron-Severi groups holds in a general setting if one assumes the Mumford-Tate conjecture.

\subsection{Mumford-Tate groups and algebraic monodromy groups of K3 surfaces.} The following result is due to Tankeev and is crucial to this paper:

Theorem 12 [Tankeev 1990; 1995]. The Mumford-Tate conjecture holds for the second cohomology group of K3 surfaces over number fields. 
This result allows us to get a Hodge-theoretic description of the Galois action on the second cohomology group of a K3 surface.

Let us now recall the description due to Zarhin [1983] of the Mumford-Tate group of a $\mathrm{K} 3$ surface. Let $X$ be a K3 surface over $\mathbb{C}$, and consider the singular cohomology $H=H^{2}(X, \mathbb{Q})$ endowed with its weight-2 Hodge structure. The Hodge structure $H$ splits as a direct sum

$$
H=N S(X) \oplus T,
$$

where $N S(X)$ is the Néron-Severi group of $X$ with rational coefficients and $T$ is the orthogonal of $N S(X)$ in $H$ with respect to the cup-product. The Hodge structure $T$ is called the transcendental part of $H^{2}(X, \mathbb{Q})$.

The Hodge structure $T$ is simple. By Lefschetz's theorem on $(1,1)$ classes, $T$ is the smallest sub-Hodge structure of $H$ such that $T \otimes \mathbb{C}$ contains $H^{2}\left(X, \mathscr{O}_{X}\right)$. By the Hodge index theorem, cup-product on $H^{2}(X, \mathbb{Q})$ restricts to a polarization $\psi: T \otimes T \rightarrow \mathbb{Q}$ on $T$.

Since $N S(X)$ is spanned by Hodge classes, the Mumford-Tate group of $H$ acts by a character on $N S(X)$ and identifies with the Mumford-Tate group of $T$. Since $T$ is polarized by $\psi, M T(T)$ is contained in the group of orthogonal similitudes $G O(T, \psi)$.

Let $E$ be the algebra of endomorphisms of the Hodge structure $T$. Zarhin [1983, Theorem 1.5.1] proves that $E$ is either a totally real field or a CM field. The field $E$ is equipped with an involution induced by the polarization on $T$, which is either the identity if $E$ is totally real or complex conjugation in case $E$ is CM.

Since $E$ consists of endomorphisms of Hodge structures, the Mumford-Tate group of $T$ commutes with $E$. By the discussion above, the Mumford-Tate group of $T$ is a subgroup of the centralizer of $E$ in the group $G O(T, \psi)$.

Theorem 13 [Zarhin 1983, Theorem 2.2.1]. The Mumford-Tate group of $T$ is the centralizer of $E$ in the group of orthogonal similitudes $G O(T, \psi)$.

Now keep the same notation, and assume $X$ can be defined over a number field $k$. Fix a prime number $\ell$. The action of the absolute Galois group $G_{k}$ on $H^{2}\left(X, \mathbb{Q}_{\ell}\right)$ leaves the $\mathbb{Q}_{\ell}$-span of the Néron-Severi group of $X$ globally invariant as well as its orthogonal complement $T_{\ell}=T \otimes \mathbb{Q}_{\ell}$. As above, the identity component of the algebraic monodromy group $G_{\ell}$ of $H^{2}\left(X, \mathbb{Q}_{\ell}\right)$ identifies with the algebraic monodromy group of $T_{\ell}$.

The polarization $\psi$ on $T$ extends to a symmetric bilinear form $\psi_{\ell}$. The representation of $G_{k}$ in the automorphism group of $T_{\ell}$ factors through the group $G O\left(T_{\ell}, \psi_{\ell}\right)$.

Since Hodge cycles on products of K3 surfaces are absolute Hodge [Deligne et al. 1982, Chapter I, 6.26], the field $E$ corresponding to endomorphisms of the 
Hodge structure $T$ acts on $T_{\ell}$ and commutes with a finite-index subgroup of $G_{k}$. As a consequence, the identity component of $G_{\ell}$ commutes with the action of $E \otimes \mathbb{Q}_{\ell}$.

By Theorem 12, the Mumford-Tate conjecture holds for $X$. As an immediate corollary of Theorem 13, we get the following description of the identity component of the algebraic monodromy group of $X$ :

Corollary 14. With notation as above, the identity component of the algebraic monodromy group associated to $T_{\ell}$ is the centralizer of $E \otimes \mathbb{Q}_{\ell}$ in the group of orthogonal similitudes $G O\left(T_{\ell}, \psi_{\ell}\right)$.

\section{Picard numbers of specializations}

This section is devoted to the proof of Theorem 1. We start by the following result, which encompasses the elementary linear algebra needed in Theorem 1.

Let $T$ be a finite-dimensional vector space endowed with a nondegenerate symmetric bilinear form $\psi$. If $f$ is any linear endomorphism of $T$, let $f^{\prime}$ be the adjoint of $f$ with respect to $\psi$.

Let $E$ be a number field acting on $T$. Assume that $E$ is stable under $e \mapsto e^{\prime}$ and that $E$ is either a totally real field with $e=e^{\prime}$ for all $e \in E$ or a CM field such that $e \mapsto e^{\prime}$ acts as complex conjugation on $E$.

Let $H$ be the centralizer of $E$ in the special orthogonal group $S O(T, \psi)$. Let $\ell$ be a prime number, and let $H_{\ell}=H \otimes \mathbb{Q}_{\ell}$.

Proposition 15. (1) If $E$ is a CM field or the dimension of $T$ as an $E$-vector space is even, then there exists $h \in H_{\ell}$ such that $h$ does not have any root of unity as an eigenvalue.

(2) If $E$ is a totally real field and the dimension of $T$ as an E-vector space is odd, then the eigenspace of any $h \in H_{\ell}$ associated to the eigenvalue 1 is of dimension at least $[E: \mathbb{Q}]$. Furthermore, there exists $h \in H_{\ell}$ for which this dimension is exactly $[E: \mathbb{Q}]$ and such that no root of unity different from 1 appears as an eigenvalue of $h$.

Proof. Let us first assume that $E$ is a totally real field. By [Zarhin 1983, 2.1], there exists a unique $E$-bilinear form $\phi: T \times T \rightarrow E$ such that $\psi=\operatorname{Tr}_{E / \mathbb{Q}}(\phi)$. With this notation, the centralizer of $E$ in $S O(T, \psi)$ is equal, as a subgroup of $G L(T)$, to the Weil restriction $\operatorname{Res}_{E / \mathbb{Q}}\left(S O_{E}(T, \phi)\right)$, where $S O_{E}(T, \phi)$ denotes the group of orthogonal similitudes of the $E$-vector space $T$ with respect to $\phi$.

Assume furthermore that the dimension of $T$ as a vector space over $E$ is even, and let us show that there is an element $h \in H_{\ell}$ such that $h$ does not have any root of unity as an eigenvalue.

Considering an orthogonal decomposition of $T$ as an $E$-vector space endowed with the bilinear form $\phi$, we can assume $T$ is of dimension 2 over $E$. Let $h$ be an 
orthogonal automorphism of the $E$-vector space $T$ of determinant 1 that is not of finite order. Then $h$ corresponds to an element of $H_{\ell}$ with the desired property.

Now if the dimension of $T$ as a vector space over $E$ is odd, recall that any element of $S_{E}(T, \psi)$ admits 1 as an eigenvalue. It follows from the description of $H_{\ell}$ as a Weil restriction that any $h \in H_{\ell}$ has 1 as an eigenvalue and that the corresponding eigenspace is invariant under the action of $E$. As a consequence, its dimension is at least $[E: \mathbb{Q}]$. One can then argue as in the previous paragraph to conclude the proof of the proposition in this case.

Let us now assume that $E$ is a CM field. Let $e$ be an element of $E$ such that $e e^{\prime}=1$ and $e$ is not a root of unity. Then multiplication by $e$ on $T$ corresponds to an element of $H_{\ell}$ as in the theorem.

We now turn to the proof of Theorem 1. From now on, we use the notation there. Let us start with a straightforward lemma.

Lemma 16. The identity component of the algebraic monodromy group associated to $T_{\ell}(1)$ is the centralizer of $E \otimes \mathbb{Q}_{\ell}$ in the special orthogonal group $\operatorname{SO}\left(T_{\ell}, \psi_{\ell}\right)$.

Proof. The representation of $G_{k}$ on $T_{\ell}(1)$ is equal to the representation of $G_{k}$ on $T_{\ell}$ twisted by the cyclotomic character. On the other hand, since the map

$$
T_{\ell}(1) \otimes T_{\ell}(1) \rightarrow \mathbb{Q}_{\ell}
$$

given by cup-product is $G_{k}$-equivariant, $G_{k}$ acts on $T_{\ell}(1)$ through the orthogonal group $O\left(T_{\ell}, \psi_{\ell}\right)$.

The lemma then follows from Corollary 14 and the fact that the special orthogonal group is the identity component of the orthogonal group.

Proof of Theorem 1. We use the notation of the theorem. First note that since specialization of Néron-Séveri groups is injective, the inequality $\rho_{\mathfrak{p}} \geq \rho$ always holds.

Let $F_{\mathfrak{p}}$ be the geometric Frobenius at $\mathfrak{p}$ acting on the étale cohomology group $H^{2}\left(\bar{X}_{\mathfrak{p}}, \mathbb{Q}_{\ell}(1)\right)$, where $\ell$ is a prime number prime to $\mathfrak{p}$. By the smooth base change theorem, the group $H^{2}\left(\bar{X}_{\mathfrak{p}}, \mathbb{Q}_{\ell}(1)\right)$ identifies with $H^{2}\left(\bar{X}, \mathbb{Q}_{\ell}(1)\right)$, and $F_{\mathfrak{p}}$ leaves both the Néron-Severi group and $T_{\ell}(1)$ globally invariant.

Let $H$ be the centralizer of $E \otimes \mathbb{Q}_{\ell}$ in the special orthogonal group $S O\left(T_{\ell}, \psi_{\ell}\right)$. Let $n$ be the dimension of $T$ as a vector space over $\mathbb{Q}$, and let $S$ be the finite set of complex roots of unity of degree at most $n$ over $\mathbb{Q}$.

Assume first that $E$ is a CM field or $E$ is a totally real field and the dimension of $T$ as a vector space over $E$ is even. By Proposition 15, the set of $h \in H_{\ell}$ such that $h$ does not have any eigenvalue in $S$ is a dense, Zariski-open subset $V_{\ell}$ of $H_{\ell}$.

By Lemma 16 and Chebotarev's density theorem, we can find a finite extension $k^{\prime}$ of $k$ with the property that if $U$ is the set of finite places $\mathfrak{p}$ of $k^{\prime}$ such that for any $\mathfrak{p} \in U, X$ has good reduction at $\mathfrak{p}$ and the geometric Frobenius $F_{\mathfrak{p}}$ acting on $T_{\ell}(1)$ does not have any eigenvalue in $S$, then $U$ has density 1 . 
Indeed, let $k^{\prime}$ be a finite extension of $k$ such that the image of absolute Galois group $G_{k^{\prime}}$ in the automorphism group of $T_{\ell}(1)$ lands in $H_{\ell}$. By Lemma 16, the image of $G_{k^{\prime}}$ is Zariski-dense in $H_{\ell}$. By [Serre 1998, I.8, Théorème 2], this implies that the set $U$ of finite places $\mathfrak{p}$ of $k^{\prime}$ such that for any $\mathfrak{p} \in U, X$ has good reduction at $\mathfrak{p}$ and the geometric Frobenius $F_{\mathfrak{p}}$ acting on $T_{\ell}(1)$ belongs to $V_{\ell}$, has density 1 .

Choose $U$ as above, and let $\mathfrak{p}$ be in $U$. By the Weil conjectures, the characteristic polynomial of the geometric Frobenius $F_{\mathfrak{p}}$ has rational coefficients. By definition of $S$, this implies that it does not have any eigenvalue that is a root of unity.

As a consequence, $F_{\mathfrak{p}}$ acting on the whole cohomology group $H^{2}\left(\bar{X}, \mathbb{Q}_{\ell}(1)\right)$ admits 1 as an eigenvalue of multiplicity $\rho$ and does not have any other eigenvalue that is a root of unity. It follows that $\rho_{\mathfrak{p}} \leq \rho$ and finally that $\rho_{\mathfrak{p}}=\rho$. This proves the first part of Theorem 1.

Now assume that $E$ is a totally real field and that the dimension of $T$ as a vector space over $E$ is odd. By Proposition 15, every element of $H_{\ell}$ has 1 as an eigenvalue with multiplicity at least $[E: \mathbb{Q}]$. By definition of the algebraic monodromy group, if $\mathfrak{p}$ is a finite place of $k$, then some power of the geometric Frobenius belongs to $H_{\ell}$.

By work of Nygaard [1983] and Nygaard and Ogus [1985] in the finite height case and the author in the supersingular case [Charles 2013], K3 surfaces over finite fields of characteristic at least 5 satisfy the Tate conjecture; namely, their geometric Picard number is equal to the dimension of the largest subspace of the second cohomology group where the Frobenius morphism acts by roots of unity. It follows that $\rho_{\mathfrak{p}} \geq \rho+[E: \mathbb{Q}]$.

By Proposition 15 again, the set of $h \in H_{\ell}$ such that $h$ admits 1 as an eigenvalue of multiplicity $[E: \mathbb{Q}]$ and does not have any other eigenvalue in $S$ is a dense, Zariski-open subset of $H_{\ell}$.

By Lemma 16 and Chebotarev's density theorem, we can find a finite extension $k^{\prime}$ of $k$ and a set $U$ of finite places $\mathfrak{p}$ of $k^{\prime}$ that has density 1 such that for any $\mathfrak{p} \in U, X$ has good reduction at $\mathfrak{p}$ and the geometric Frobenius $F_{\mathfrak{p}}$ acting on $T_{\ell}(1)$ admits 1 as an eigenvalue of multiplicity $[E: \mathbb{Q}]$ and does not have any other eigenvalue in $S$.

Choose $U$ as above, and let $\mathfrak{p}$ be in $U$. Since $X_{\mathfrak{p}}$ satisfies the Tate conjecture, we can argue as above to finish the proof of Theorem 1.

Remark 17. Using Frobenius tori as in [Serre 1981] and the fact that Frobenius tori are maximal tori of the Mumford-Tate groups for infinitely many primes, one can work directly in the group of orthogonal similitudes instead of reducing to the special orthogonal group as in Lemma 16.

\section{Discriminants of Néron-Severi groups}

In this section, we discuss properties of the Néron-Severi lattices of specializations of K3 surfaces. Once again, we use the notation of Theorem 1. 
Proposition 18. Assume that $E$ is a totally real field and that the dimension of $T$ over $E$ is odd. If $\mathfrak{p}$ is a finite place of $k$ such that $X$ has good reduction at $\mathfrak{p}$, denote by $\delta(\mathfrak{p}) \in \mathbb{Q}^{*} /\left(\mathbb{Q}^{*}\right)^{2}$ the discriminant of the lattice $N S\left(\bar{X}_{\mathfrak{p}}\right)$ with respect to the intersection product.

There exist infinitely many finite places $\mathfrak{p}_{i}, i \in \mathbb{N}$, and infinitely many finite places $\mathfrak{q}_{j}, j \in \mathbb{N}$, of $k$ such that for any integers $i$ and $j$

(1) X has good, ordinary reduction at both $\mathfrak{p}_{i}$ and $\mathfrak{q}_{j}$,

(2) $\rho_{\mathfrak{p}_{i}}=\rho_{\mathfrak{q}_{j}}=\rho+[E: \mathbb{Q}]$ and

(3) $\delta\left(\mathfrak{p}_{i}\right) \neq \delta\left(\mathfrak{q}_{j}\right)$.

Remark 19. A special case of this result is that the method developed in [van Luijk 2007] to prove that a given K 3 surface over a number field has Picard number 1 always works in the case $E=\mathbb{Q}$. We noted in Remark 3 that it cannot work directly otherwise.

In Section 5, we will adapt the method so as to make it work in every case.

We start with some easy linear algebra.

Lemma 20. Let $\ell$ be a prime number, and let $V$ be a free module of finite rank over $\mathbb{Z}_{\ell}$. Let $g$ be an endomorphism of $V$ such that $g \otimes \mathbb{Q}_{\ell}$ is a semisimple automorphism of $V \otimes \mathbb{Q}_{\ell}$, and denote by $r$ the multiplicity of 1 as an eigenvalue of $g$. Let $W$ be the eigenspace associated to the eigenvalue 1 of $g$. Let $d$ be a positive integer.

Then there exists an integer $N$ with the following property. Let $h$ be an endomorphism of $V$ such that $h \otimes \mathbb{Q}_{\ell}$ is a semisimple automorphism of $V \otimes \mathbb{Q}_{\ell}$. Assume that $r$ is the multiplicity of 1 as an eigenvalue of $h$, and let $W^{\prime}$ be the eigenspace associated to the eigenvalue 1 of $h$. If $h$ is congruent to $g$ modulo $\ell^{N}$, then $W \otimes \mathbb{Z} / \ell^{d} \mathbb{Z}=W^{\prime} \otimes \mathbb{Z} / \ell^{d} \mathbb{Z}$.

Remark 21. In particular, if $V$ is endowed with a symmetric bilinear form $\phi$ such that the restriction of $\phi$ to $W$ is not degenerate and $N$ is sufficiently large, then the discriminants of $W$ and $W^{\prime}$ are equal in $\mathbb{Q}_{\ell}^{*} /\left(\mathbb{Q}_{\ell}^{*}\right)^{2}$.

Proof. Write $V=W \oplus \widetilde{W}$, where $\widetilde{W}$ is a $g$-invariant submodule of $V$. Since $g \otimes \mathbb{Q}_{\ell}$ does not fix any nonzero element of $\widetilde{W} \otimes \mathbb{Q}_{\ell}$, a compactness argument shows that there exists an integer $N$ such that if $g(v)-v \in l^{N} V$ for some $v \in \widetilde{W}$, then $v \in \ell^{k} \widetilde{W}$.

Let $h$ be as in the statement of the lemma. By definition of $N$, if $v \in V$ is fixed by $h$, then $v \otimes \mathbb{Z} / \ell^{k} \mathbb{Z} \in W \otimes \mathbb{Z} / \ell^{k} \mathbb{Z}$. With the notation of the lemma, it follows that $W^{\prime} \otimes \mathbb{Z} / \ell^{k} \mathbb{Z} \subset W \otimes \mathbb{Z} / \ell^{k} \mathbb{Z}$. Since both $W$ and $W^{\prime}$ are saturated submodules of $V$ of the same rank $r$, equality follows.

Proof of Proposition 18. First note that the dimension of $T$ as a vector space over $E$ is at least 3. Indeed, let $\omega$ be a generator of $T^{2,0} \subset T \otimes \mathbb{C}$, and let $\sigma: E \rightarrow \mathbb{C}$ be 
the complex embedding of $E$ satisfying

$$
e . \omega=\sigma(e) \omega \quad \text { for all } e \in E .
$$

The complex lines $\mathbb{C} \omega$ and $\mathbb{C} \bar{\omega}$ are two distinct one-dimensional subspaces of $T_{E} \otimes_{\sigma} \mathbb{C}$, where $T_{E}$ denotes $T$ endowed with the structure of a vector space over $E$. As a consequence, the dimension of $T$ as a vector space over $E$ is at least 2 and at least 3 since we assumed it to be odd.

Recall that $\psi$ is the bilinear form on $T$ induced by cup-product. As in Proposition 15, there exists a unique $E$-bilinear form $\phi: T \times T \rightarrow E$ such that $\psi=\operatorname{Tr}_{E / \mathbb{Q}}(\phi)$. Any orthogonal basis of $T_{E}$ with respect to $\phi$ induces an orthogonal decomposition of $T$ with respect to $\psi$

$$
T=T_{1} \oplus \cdots \oplus T_{r},
$$

where the $T_{i}$ are stable under the action of $E$ and of dimension 1 as $E$-vector spaces.

By the same reasoning as above, since the $T_{i}$ are one-dimensional over $E$, there is no integer $i$ such that $T_{i} \otimes \mathbb{C}$ contains the two-dimensional space $T^{2,0} \oplus T^{0,2}$.

The signature of $\psi$ on $T$ is $(2, \operatorname{dim}(T)-2)$. By the Hodge index theorem and the remark above, the signature of the restriction of $\psi$ to $T_{i}$ is either $(0,[E: \mathbb{Q}])$ or $(1,[E: \mathbb{Q}]-1)$. Since the dimension of $T$ over $E$ is at least 3 , both these signatures appear, and this implies that, up to reordering, we can assume that the discriminant of $T_{1}$ is negative and the discriminant of $T_{2}$ is positive. Let $\delta$ and $\delta^{\prime}$ be these two discriminants in $\mathbb{Q}^{*} /\left(\mathbb{Q}^{*}\right)^{2}$.

Since $\delta \neq \delta^{\prime}$ in $\mathbb{Q}^{*} /\left(\mathbb{Q}^{*}\right)^{2}$, there exists a prime number $\ell$ such that the images of $\delta$ and $\delta^{\prime}$ in $\mathbb{Q}_{\ell}^{*} /\left(\mathbb{Q}_{\ell}^{*}\right)^{2}$ are different. If $W$ is any subspace of $T_{\ell}$ such that the restriction of $\psi_{\ell}$ to $W$ is nondegenerate, let $\delta(W)$ denote the discriminant of $W$ in $\mathbb{Q}_{\ell}^{*} /\left(\mathbb{Q}_{\ell}^{*}\right)^{2}$

By Lemma 20, Proposition 15 and Chebotarev's density theorem, we can find, for any positive integer $d$, infinitely many finite places $\mathfrak{p}_{i}, i \in \mathbb{N}$, and infinitely many finite places $\mathfrak{q}_{j}, j \in \mathbb{N}$, of $k$ such that for any integers $i$ and $j$

(1) $X$ has good, ordinary reduction at both $\mathfrak{p}_{i}$ and $\mathfrak{q}_{j}$,

(2) $\rho_{\mathfrak{p}_{i}}=\rho_{\mathfrak{q}_{j}}=\rho+[E: \mathbb{Q}]$,

(3) if $F_{\mathfrak{p}_{i}}$ and $F_{\mathfrak{q}_{j}}$ denote the geometric Frobeniuses at $\mathfrak{p}_{i}$ and $\mathfrak{q}_{j}$ acting on $T_{\ell}(1)$ and $W_{\mathfrak{p}_{i}}$ and $W_{\mathfrak{q}_{j}}$ denote the eigenspaces associated to the eigenvalue 1 of $F_{\mathfrak{p}_{i}}$ and $F_{\mathfrak{q}_{j}}$, then $W_{\mathfrak{p}_{i}} \otimes \mathbb{Z} / \ell^{d} \mathbb{Z}=T_{1} \otimes \mathbb{Z} / \ell^{d} \mathbb{Z}$ and $W_{\mathfrak{q}_{j}} \otimes \mathbb{Z} / \ell^{d} \mathbb{Z}=T_{2} \otimes \mathbb{Z} / \ell^{d} \mathbb{Z}$, respectively, and

(4) the geometric Frobeniuses $F_{\mathfrak{p}_{i}}$ and $F_{\mathfrak{q}_{j}}$ acting on $T_{\ell}(1)$ do not have any eigenvalue different from 1 that is a root of unity.

If $d$ is big enough, this implies that $\delta\left(W_{\mathfrak{p}_{i}}\right)=\delta$ and $\delta\left(W_{\mathfrak{q}_{j}}\right)=\delta^{\prime}$ in $\mathbb{Q}_{\ell}^{*} /\left(\mathbb{Q}_{\ell}^{*}\right)^{2}$. Proposition 18 immediately follows by the Tate conjecture for ordinary K3 surfaces. 
Remark 22. The proof above shows that the density of pairs $(\mathfrak{p}, \mathfrak{q})$ as in the proposition is positive.

\section{Computing the Picard number over number fields}

This section is devoted to a proof of Theorem 5. Given a projective K3 surface over a number field $k$, we want to compute the Picard number of $X$ using the equations of $X$ in a projective embedding.

There are two steps in our approach. The first one is finding sufficiently many divisors on $X$, and the second is proving that the rational span of these divisors is the whole Néron-Severi group of $X$.

In case we want to prove that the K3 surface has Picard number 1, the first step is vacuous as we already have a divisor given by a hyperplane section. In general, the first step is done by going through the Hilbert schemes of curves in the projective space we are working in and doing elimination theory to find curves on $X$. After a finite number of computations, this will allow us to find divisors on $X$ that span the Néron-Severi group.

The second step will be done by reducing to finite characteristic and using our results above.

However, this is not sufficient. Indeed, the field $E$ of endomorphisms of the transcendental part of the Hodge structure of $X$ plays a role in the behavior of the Picard number after specialization, and in case $E$ is a totally real field strictly containing $\mathbb{Q}$ such that $T$ is of odd dimension over $E$, this leads to some loss of accuracy in the estimates reduction at finite places can provide.

This problem will be solved by studying codimension-2 varieties in $X \times X$. Assuming the Hodge conjecture for $X \times X$, these determine the field $E$, which will allow us to conclude.

We start by the following result:

Proposition 23. Let $X$ be a $K 3$ surface over a number field $k$. Assume we are given the equations of $X$ in some projective embedding.

Let $T$ be the transcendental part of $H^{2}(X, \mathbb{Q})$, and let $E$ be the field of endomorphisms of the Hodge structure T.

Assume that we know that the Picard number of $X$ is greater or equal than some integer $\rho$ and that the degree of $E$ over $\mathbb{Q}$ is greater or equal than some integer $d$. Then there exists an algorithm with the following properties:

(1) If the algorithm terminates, it proves that the Picard number of $X$ is $\rho$.

(2) Suppose that the Picard number of $X$ is actually $\rho$. Then the algorithm terminates unless $E$ is totally real, the dimension of $T$ as a vector space over $E$ is odd and $d<[E: \mathbb{Q}]$. 
Proof. Let $\rho^{\prime}$ be the actual Picard number of $X$. We know that $\rho^{\prime} \geq \rho$. Using the Weil conjectures [Deligne 1974], we can compute the characteristic polynomial of Frobenius at any finite place $\mathfrak{p}$ of $k$ of good reduction; see [van Luijk 2007; Elsenhans and Jahnel 2012]. This allows in particular to compute the numbers $\rho_{\mathfrak{p}}$. Using the Artin-Tate formula [Tate 1966, Conjecture (C)], which holds for surfaces satisfying the Tate conjecture by [Milne 1975], one can also compute the discriminants $\delta(\mathfrak{p})$ as in Proposition 18.

We start computing $\rho_{\mathfrak{p}}$ and $\delta(\mathfrak{p})$ for all places $\mathfrak{p}$ of good reduction.

Let us distinguish three cases. First assume that $E$ is a CM field. By Theorem 1, we can find $\mathfrak{p}$ with $\rho_{\mathfrak{p}}=\rho^{\prime}$. If it happens that $\rho$, the lower bound for the Picard number of $X$ that we were given, is equal to the actual Picard number $\rho^{\prime}$ (that we do not know yet), the computation at $\mathfrak{p}$ together with this lower bound allows us to prove that $X$ has Picard number $\rho=\rho^{\prime}$.

Now assume that $E$ is totally real and the dimension of $T$ as a vector space over $E$ is even. In that case, Theorem 1 allows us to make the same conclusion.

The last case happens when $E$ is totally real and the dimension of $T$ as a vector space over $E$ is odd. By Proposition 18, the finite field computations give us two finite places $\mathfrak{p}$ and $\mathfrak{q}$ of $k$ where $X$ has good, ordinary reduction, with $\rho_{\mathfrak{p}}=\rho_{\mathfrak{q}}=\rho^{\prime}+[E: \mathbb{Q}]$ and $\delta(\mathfrak{p}) \neq \delta(\mathfrak{q})$.

Since $\delta(\mathfrak{p}) \neq \delta(\mathfrak{q})$, we know that the specialization maps $N S(\bar{X}) \rightarrow N S\left(\bar{X}_{\mathfrak{p}}\right)$ and $N S(\bar{X}) \rightarrow N S\left(\bar{X}_{\mathfrak{p}}\right)$ are not surjective. This means that $N S\left(X_{\mathfrak{p}}\right) \cap T_{\ell}(1)$ is nonzero in $H^{2}\left(\bar{X}_{\mathfrak{p}}, \mathbb{Q}_{\ell}(1)\right)$.

Now we know by the analysis in the proof of Theorem 1 that this intersection is stable under the action of $E$. As a consequence, its dimension is at least $[E: \mathbb{Q}] \geq d$. This gives us the estimation

$$
\rho^{\prime} \leq \rho_{\mathfrak{p}}-d
$$

In case $d$ happens to be equal to the actual degree $[E: \mathbb{Q}]$ and $\rho=\rho^{\prime}$, these estimates allow us to prove that $X$ has Picard number $\rho=\rho^{\prime}$.

Remark 24. In case $\rho=\rho^{\prime}=1$ and $E=\mathbb{Q}$, this proves that the method of [van Luijk 2007] always works.

Proof of Theorem 5. Let $X, E$ and $T$ be as above. Let $\rho^{\prime}$ be the Picard number of $X$ and $d^{\prime}$ the degree of $E$ over $\mathbb{Q}$. By Proposition 23, we only need to be able to prove that the Picard number of $X$ is at least $\rho^{\prime}$ and the degree of $E$ over $\mathbb{Q}$ is at least $d^{\prime}$.

The assertion on the Picard number is theoretically - although not computationally - easy. One can go through Hilbert schemes of curves in the projective space where $X$ is given and check, using elimination theory, for curves that happen to lie on $X$. Computing intersection matrices with these divisors on $X$, one can find divisors that span a $\rho^{\prime}$-dimensional subset of the Néron-Severi group of $X$. 
Running these Hilbert scheme computations alongside the computations of Proposition 23 allows for a computation of the Picard number of $X$ unless $E$ is a totally real field strictly containing $\mathbb{Q}$ such that $T$ is of odd dimension over $E$.

To deal with the latter case, one has to work on $X \times X$. If one assumes the Hodge conjecture for $X \times X$, then elements of $E$ are induced by codimension- 2 cycles in $X \times X$. As above, one can use Hilbert schemes to find codimension-2 subschemes in $X \times X$.

Given such a subscheme $Z$, the action of $Z$ on $T$ can be determined by first computing the characteristic polynomial of the correspondence $H^{2}(X, \mathbb{Q}) \rightarrow H^{2}(X, \mathbb{Q})$ by computing intersection numbers between $T$ and the various subschemes obtained by composing the correspondence induced by $Z$ with itself.

Factoring the characteristic polynomial, this gives candidates for the algebraic number $\lambda$ such that $[Z]_{*} \eta=\lambda \eta$, where $\eta$ is a nonzero algebraic 2-form on $X$. An approximate computation can then determine $\lambda$. The degree of $\lambda$ over $\mathbb{Q}$ is a lower bound for $[E: \mathbb{Q}]$.

By the primitive element theorem, it is easy to see that one can find $Z$ such that this computation gives an optimal estimate for the degree of $E$. Using Proposition 23, this concludes the proof.

In conclusion, an algorithm to compute Picard number of K3 surfaces works as follows. Let $X$ be a K3 surface. Run the three algorithms alongside each other:

(1) Going through Hilbert schemes of a suitable projective space, find divisors on $X$ and compute the dimension of their span in the Néron-Severi group via intersection theory. This gives a lower bound for the Picard number.

(2) Going through Hilbert schemes of a suitable projective space, find codimension2 cycles in $X \times X$. Using intersection theory again, use these to get a lower bound on the field $E$ of endomorphisms of the transcendental part of $H^{2}(X, \mathbb{Q})$.

(3) Going through finite places $\mathfrak{p}$ of $k$, compute the Picard number and the discriminant of the Néron-Severi group of $\bar{X}_{\mathfrak{p}}$ by counting points over finite fields. Using the preceding step, get an upper bound on the Picard number of $X$.

We showed that the estimates provided by the method solve the problem unconditionally unless $E$ is a totally real field strictly containing $\mathbb{Q}$ and the transcendental part of $H^{2}(X, \mathbb{Q})$ is of odd dimension over $E$. In the latter case, the estimates above are sufficiently precise to compute the Picard number if we assume the Hodge conjecture for $X \times X$.

Remark 25. It seems that the computations of the second step above would be very lengthy to do in practice. We however wanted to point out that they can be done.

Note that the computations terminate much faster in most cases since $E=\mathbb{Q}$ for the majority of $\mathrm{K} 3$ complex surfaces in the sense of Baire category. 


\section{Acknowledgments}

This paper was written during a stay at the University of Bonn. I would like to thank Daniel Huybrechts for his hospitality and many useful comments. I thank Bjorn Poonen and Matthias Schuett for useful remarks on a first version of this text.

\section{References}

[André 1996] Y. André, "Pour une théorie inconditionnelle des motifs", Inst. Hautes Études Sci. Publ. Math. 83 (1996), 5-49. MR 98m:14022 Zbl 0874.14010

[Bogomolov, Hassett and Tschinkel 2011] F. Bogomolov, B. Hassett, and Y. Tschinkel, "Constructing rational curves on K3 surfaces", Duke Math. J. 157:3 (2011), 535-550. MR 2012d:14061 Zbl 1236.14035

[Charles 2013] F. Charles, "The Tate conjecture for K3 surfaces over finite fields", Invent. Math. 194:1 (2013), 119-145. MR 3103257 Zbl 1282.14014

[Deligne 1974] P. Deligne, "La conjecture de Weil, I", Inst. Hautes Études Sci. Publ. Math. 43 (1974), 273-307. MR 49 \#5013 Zbl 0287.14001

[Deligne et al. 1982] P. Deligne, J. S. Milne, A. Ogus, and K.-y. Shih, Hodge cycles, motives, and Shimura varieties, Lecture Notes in Mathematics 900, Springer, Berlin, 1982. MR 84m:14046 Zbl 0465.00010

[Elsenhans and Jahnel 2008a] A.-S. Elsenhans and J. Jahnel, "K3 surfaces of Picard rank one and degree two", pp. 212-225 in Algorithmic number theory, edited by A. J. van der Poorten and A. Stein, Lecture Notes in Comput. Sci. 5011, Springer, Berlin, 2008. MR 2010h:11102 Zbl 1205.11073

[Elsenhans and Jahnel 2008b] A.-S. Elsenhans and J. Jahnel, "K3 surfaces of Picard rank one which are double covers of the projective plane", pp. 63-77 in Higher-dimensional geometry over finite fields, edited by D. Kaledin and Y. Tschinkel, NATO Sci. Peace Secur. Ser. D Inf. Commun. Secur. 16, IOS, Amsterdam, 2008. MR 2009j:14047 Zbl 1182.14036

[Elsenhans and Jahnel 2012] A.-S. Elsenhans and J. Jahnel, "Kummer surfaces and the computation of the Picard group", LMS J. Comput. Math. 15 (2012), 84-100. MR 2911278

[van Geemen 2008] B. van Geemen, "Real multiplication on K3 surfaces and Kuga-Satake varieties", Michigan Math. J. 56:2 (2008), 375-399. MR 2009k:14073 Zbl 1161.14027

[Hassett and Várilly-Alvarado 2013] B. Hassett and A. Várilly-Alvarado, "Failure of the Hasse principle on general K3 surfaces”, J. Inst. Math. Jussieu 12:4 (2013), 853-877. MR 3103134

[Hassett et al. 2011] B. Hassett, A. Várilly-Alvarado, and P. Varilly, “Transcendental obstructions to weak approximation on general K3 surfaces”, Adv. Math. 228:3 (2011), 1377-1404. MR 2012i:14025 Zbl 1228.14030

[Hassett et al. 2013] B. Hassett, A. Kresch, and Y. Tschinkel, "Effective computation of Picard groups and Brauer-Manin obstructions of degree two K3 surfaces over number fields", Rend. Circ. Mat. Palermo (2) 62:1 (2013), 137-151. MR 3031574

[de Jong and Katz 2000] A. J. de Jong and N. M. Katz, "Monodromy and the Tate conjecture: Picard numbers and Mordell-Weil ranks in families", Israel J. Math. 120:part A (2000), 47-79. MR 2002b:14026 Zbl 1067.14504

[Li and Liedtke 2012] J. Li and C. Liedtke, "Rational curves on K3 surfaces", Invent. Math. 188:3 (2012), 713-727. MR 2917181 Zbl 1255.14026

[van Luijk 2007] R. van Luijk, "K3 surfaces with Picard number one and infinitely many rational points”, Algebra Number Theory 1:1 (2007), 1-15. MR 2008d:14058 Zbl 1123.14022 
[Maulik and Poonen 2012] D. Maulik and B. Poonen, "Néron-Severi groups under specialization", Duke Math. J. 161:11 (2012), 2167-2206. MR 2957700 Zbl 1248.14011

[Milne 1975] J. S. Milne, "On a conjecture of Artin and Tate”, Ann. of Math. (2) 102:3 (1975), 517-533. MR 54 \#2659 Zbl 0343.14005

[Mukai 2002] S. Mukai, "Vector bundles on a K3 surface", pp. 495-502 in Proceedings of the International Congress of Mathematicians, Vol. II (Beijing, 2002), edited by T. Li, Higher Ed. Press, Beijing, 2002. MR 2004b:14067 Zbl 1046.14016

[Nygaard 1983] N. O. Nygaard, "The Tate conjecture for ordinary K3 surfaces over finite fields", Invent. Math. 74:2 (1983), 213-237. MR 85h:14012 Zbl 0557.14002

[Nygaard and Ogus 1985] N. Nygaard and A. Ogus, "Tate's conjecture for K3 surfaces of finite height”, Ann. of Math. (2) 122:3 (1985), 461-507. MR 87h:14014 Zbl 0591.14005

[Pink 1998] R. Pink, "l-adic algebraic monodromy groups, cocharacters, and the Mumford-Tate conjecture", J. Reine Angew. Math. 495 (1998), 187-237. MR 98m:11060 Zbl 0920.14006

[Serre 1981] J.-P. Serre, Letter to K. Ribet of January 1, 1981. Printed in his Euvres, vol. IV: 1985-1998, pp. 1-17, Springer, Berlin, 1986.

[Serre 1998] J.-P. Serre, Abelian l-adic representations and elliptic curves, Research Notes in Mathematics 7, A K Peters Ltd., Wellesley, MA, 1998. Revised reprint of the 1968 original. MR 98g:11066 Zbl 0902.14016

[Shioda 1981] T. Shioda, "On the Picard number of a complex projective variety", Ann. Sci. École Norm. Sup. (4) 14:3 (1981), 303-321. MR 83i:14005 Zbl 0498.14018

[Shioda 1983] T. Shioda, "Algebraic cycles on a certain hypersurface", pp. 271-294 in Algebraic geometry (Tokyo and Kyoto, 1982), edited by M. Raynaud and T. Shioda, Lecture Notes in Math. 1016, Springer, Berlin, 1983. MR 85d:14015 Zbl 0579.14005

[Tankeev 1990] S. G. Tankeev, "Surfaces of K3 type over number fields and the Mumford-Tate conjecture”, Izv. Akad. Nauk SSSR Ser. Mat. 54:4 (1990), 846-861. In Russian; translated in Math. USSR-Izv. 37:1 (1991), 191-208. MR 91m:11044

[Tankeev 1995] S. G. Tankeev, "Surfaces of K3 type over number fields and the Mumford-Tate conjecture. II”, Izv. Ross. Akad. Nauk Ser. Mat. 59:3 (1995), 179-206. In Russian; translated in Izv. Math. 59:3 (1995), 619-646. MR 97d:11104 Zbl 0895.14011

[Tate 1966] J. Tate, "On the conjectures of Birch and Swinnerton-Dyer and a geometric analog", exposé no. 306 in Séminaire Bourbaki 1965/66, Benjamin, New York, 1966. Reprinted in Dix exposés sur la cohomologie des schémas, Adv. Stud. Studies Pure Math. 3, 189-214, North-Holland, Amsterdam, 1968, and in Sém. Bourbaki, vol. 9, 415-440, Soc. Mat. de France, 1995. Zbl 0199.55604

[Terasoma 1985] T. Terasoma, "Complete intersections with middle Picard number 1 defined over Q”, Math. Z. 189:2 (1985), 289-296. MR 86f:14010 Zbl 0579.14006

[Vasiu 2008] A. Vasiu, "Some cases of the Mumford-Tate conjecture and Shimura varieties", Indiana Univ. Math. J. 57:1 (2008), 1-75. MR 2010a:14082 Zbl 1173.11039

[Voisin 2002] C. Voisin, Théorie de Hodge et géométrie algébrique complexe, Cours Spécialisés 10, Société Mathématique de France, Paris, 2002. MR 2005c:32024a Zbl 1032.14001

[Zarhin 1983] Y. G. Zarhin, "Hodge groups of K3 surfaces”, J. Reine Angew. Math. 341 (1983), 193-220. MR 84g:14009

Communicated by János Kollár

Received 2012-02-01 Revised 2012-09-20 Accepted 2012-11-04

francois.charles@univ-rennes1.fr Institut de Recherche Mathématiques de Rennes, Université de Rennes 1, Campus de Beaulieu, 35042 Rennes, France http://perso.univ-rennes1.fr/francois.charles/ 


\section{Algebra \& Number Theory}

msp.org/ant

\section{EDITORS}

MANAGING EDITOR

Bjorn Poonen

Massachusetts Institute of Technology

Cambridge, USA

\author{
EDITORIAL BOARD CHAIR \\ David Eisenbud \\ University of California \\ Berkeley, USA
}

\section{BOARD OF EDITORS}

Georgia Benkart

Dave Benson

Richard E. Borcherds

John H. Coates

J-L. Colliot-Thélène

Brian D. Conrad

Hélène Esnault

Hubert Flenner

Edward Frenkel

Andrew Granville

Joseph Gubeladze

Roger Heath-Brown

Ehud Hrushovski

Craig Huneke

Mikhail Kapranov

Yujiro Kawamata

János Kollár

Yuri Manin

Barry Mazur

Philippe Michel
University of Wisconsin, Madison, USA

University of Aberdeen, Scotland

University of California, Berkeley, USA

University of Cambridge, UK

CNRS, Université Paris-Sud, France

University of Michigan, USA

Freie Universität Berlin, Germany

Ruhr-Universität, Germany

University of California, Berkeley, USA

Université de Montréal, Canada

San Francisco State University, USA

Oxford University, UK

Hebrew University, Israel

University of Virginia, USA

Yale University, USA

University of Tokyo, Japan

Princeton University, USA

Northwestern University, USA

Harvard University, USA

École Polytechnique Fédérale de Lausanne
Susan Montgomery

Shigefumi Mori

Raman Parimala

Jonathan Pila

Victor Reiner

Karl Rubin

Peter Sarnak

Joseph H. Silverman

Michael Singer

Vasudevan Srinivas

J. Toby Stafford

Bernd Sturmfels

Richard Taylor

Ravi Vakil

Michel van den Bergh

Marie-France Vignéras

Kei-Ichi Watanabe

Efim Zelmanov

Shou-Wu Zhang
University of Southern California, USA

RIMS, Kyoto University, Japan

Emory University, USA

University of Oxford, UK

University of Minnesota, USA

University of California, Irvine, USA

Princeton University, USA

Brown University, USA

North Carolina State University, USA

Tata Inst. of Fund. Research, India

University of Michigan, USA

University of California, Berkeley, USA

Harvard University, USA

Stanford University, USA

Hasselt University, Belgium

Université Paris VII, France

Nihon University, Japan

University of California, San Diego, USA

Princeton University, USA

PRODUCTION

production@msp.org

Silvio Levy, Scientific Editor

See inside back cover or msp.org/ant for submission instructions.

The subscription price for 2014 is US $\$ 225 /$ year for the electronic version, and $\$ 400 /$ year $(+\$ 55$, if shipping outside the US) for print and electronic. Subscriptions, requests for back issues and changes of subscribers address should be sent to MSP.

Algebra \& Number Theory (ISSN 1944-7833 electronic, 1937-0652 printed) at Mathematical Sciences Publishers, 798 Evans Hall \#3840, c/o University of California, Berkeley, CA 94720-3840 is published continuously online. Periodical rate postage paid at Berkeley, CA 94704, and additional mailing offices.

ANT peer review and production are managed by EditFLOW ${ }^{\circledR}$ from Mathematical Sciences Publishers.

\section{PUBLISHED BY}

- mathematical sciences publishers

nonprofit scientific publishing

http://msp.org/

(C) 2014 Mathematical Sciences Publishers 


\section{Algebra \& Number Theory}

\section{Volume $8 \quad$ No. $1 \quad 2014$}

On the Picard number of K3 surfaces over number fields

FRANÇOIS CHARLES

Adèle residue symbol and Tate's central extension for multiloop Lie algebras

OLIVER BRAUNLING

On the number of cubic orders of bounded discriminant having automorphism group $C_{3}$, and related problems

MANJUl Bhargava and ARIEl ShNidman

Polynomial bounds for Arakelov invariants of Belyi curves

ARIYAN JAVANPEYKAR

The radius of a subcategory of modules

HAILONG DAO and RYO TAKAHASHI

A generalized Bogomolov-Gieseker inequality for the three-dimensional projective space

EMANUELE MACRÌ

$(\varphi, \Gamma)$-modules over noncommutative overconvergent and Robba rings

GERGELY ZÁBRÁDI

The Tannakian formalism and the Langlands conjectures

DAVID KAZHDAN, MICHAEL LARSEN and YAKOV VARSHAVSKY 